\title{
Nail-Patella Syndrome: clinical and molecular data in 55 families raising the hypothesis of a genetic heterogeneity
}

\begin{abstract}
Jamal Ghoumid ${ }^{1,2,6}$, Florence Petit ${ }^{1,2,6}$, Muriel Holder-Espinasse ${ }^{1,2,3}$, Anne-Sophie Jourdain ${ }^{4}$, José Guerra ${ }^{1}$, Anne Dieux-Coeslier ${ }^{1}$, Martin Figeac ${ }^{5}$, Nicole Porchet ${ }^{2,3}$, Sylvie Manouvrier-Hanu ${ }^{\star, 1,2}$ and Fabienne Escande ${ }^{3}$

Nail-Patella Syndrome (NPS) is a rare autosomal dominant condition comprising nail and skeletal anomalies. Skeletal features include dysplastic patellae and iliac horns, as well as scapula and elbow dysplasia. Nephropathy and glaucoma or intra-ocular hypertension can sometimes be present. NPS is due to variants affecting function in LMX1B, which encodes a LIMhomeodomain protein critical for limb, kidney and eye development. We describe the phenotype and the molecular data of 55 index patients and their 39 relatives presenting with typical NPS. We identified 38 different $L M X 1 B$ anomalies, 19 of which were not reported before. In our series, $9 \%$ of families are not carriers of a $L M X 1 B$ genomic alteration after extensive study of the coding and non-coding regions of the gene. One of the families showed no linkage to the $L M X 1 B$ locus, raising the hypothesis of a genetic heterogeneity.
\end{abstract}

European Journal of Human Genetics (2016) 24, 44-50; doi:10.1038/ejhg.2015.77; published online 22 April 2015

\section{INTRODUCTION}

Nail-Patella Syndrome (NPS, MIM 161200) is a rare autosomal dominant condition characterised by variable nail, skeletal, renal and ocular anomalies. The disease was first described by Chatelain, in 1820 , in a patient with congenital nail, elbow and patellar defects (quoted by Roeckerath ${ }^{1}$ ). Further reports improved the clinical description and broadened the spectrum of associated findings. ${ }^{2-5}$ Almost all affected individuals show nail dysplasia, consisting of koilonychia, or even anonychia, usually with the ulnar side of the nail more severely affected than the radial side. Typical lunular anomalies comprise triangular or absent lunulae. The thumbnails are the most severely affected, and the severity tends to decrease towards the little finger. Skeletal features are frequently described, including patellar anomalies (hypoplasia or agenesis), iliac horns and elbow anomalies (radial head hypoplasia or dislocation). ${ }^{2}$ Besides nail and skeletal defects, $22-60 \%$ patients present with renal anomalies, ${ }^{2,4,6-9}$ extending from asymptomatic proteinuria to endstage renal failure. Ocular anomalies were first described in NPS by Lichter et al., ${ }^{5}$ corresponding to variable degree of intra-ocular hypertension $(\mathrm{IOH})$, glaucoma or normal tension glaucoma. Recently, neurological features comprising peripheral neuropathies, vasomotor disorder, hyperactivity or depression were also linked to the condition. , $^{2}$

The disease-causing gene is $L M X 1 B$ (MIM 602575), mapped on chromosome $9 \mathrm{q} 34$. It consists of eight exons spanning a region of $\approx 82 \mathrm{~kb}$ of genomic DNA, and encodes a LIM-homeodomain transcription factor involved in many developmental mechanisms and accounting for the pleiotropic manifestations of the disease. ${ }^{10,11}$ The protein comprises two LIM domains (LIM-A and LIM-B) and a homeodomain (HD). The LIM domains are composed of two zinc fingers and are involved in protein-protein interactions. The HD is a 60 -amino acid domain, highly conserved through evolution and necessary for DNA binding and transcriptional activation. ${ }^{11}$

The main pathogenic mechanism underlying autosomal dominant NPS in humans is haploinsufficiency. ${ }^{12-14}$ The majority of patients carry either $L M X 1 B$ variants or complete or partial deletions responsible for haploinsufficiency through the nonsense-mediated mRNA decay pathway. ${ }^{12}$ Missense variants located in functional domains cause diminished transcriptional activity and DNA-binding ability, thus responsible for the partial loss of function of LMX1B. ${ }^{11,13,14}$ Nevertheless, although no clear genotype-phenotype correlation was apparent for extra-renal manifestations, Bongers and co-workers ${ }^{2}$ observed that individuals with variants located in the HD had significantly higher renal involvement raising the possibility of dominant-negative and/or gain-of-function effects. Furthermore, recent studies in mice have demonstrated that missense variants of the HD can show dominant-negative activity in vivo. ${ }^{15}$

In the present study, we describe the phenotype and molecular data of 55 index patients and their 39 relatives affected with NPS. Variants or large rearrangements of $L M X 1 B$ were identified in $91 \%$ of patients. Nineteen previously unreported variants are described. In the remaining $9 \%$ of patients, no variant affecting function was identified in $L M X 1 B$ after further investigation and the hypothesis of a genetic heterogeneity is raised.

${ }^{1}$ Service de Génétique Clinique, Hôpital Jeanne de Flandre, CHRU, Lille, France; ${ }^{2}$ Faculté de Médecine, Université Lille Nord de France, Lille, France; ${ }^{3}$ Clinical Genetics Department, Guy's Hospital, London, UK; ${ }^{4}$ Laboratoire de Biologie Moléculaire, Centre de Biologie-Pathologie, CHRU, Lille, France; ${ }^{5}$ Functional and Structural Genomic Platform, Université Lille 2, Lille, France

*Correspondence: Professor S Manouvrier-Hanu, Service de Génétique Clinique Hôpital Jeanne de Flandre - CHRU, 2, Avenue Eugène Avinée, Lille, Cedex 59037, France. Tel: +33 3204449 11; Fax: +33 3204449 01; E-mail: sylvie.manouvrier@chru-lille.fr

6These authors contributed equally to this work.

Received 26 October 2014; revised 18 February 2015; accepted 10 March 2015; published online 22 April 2015 


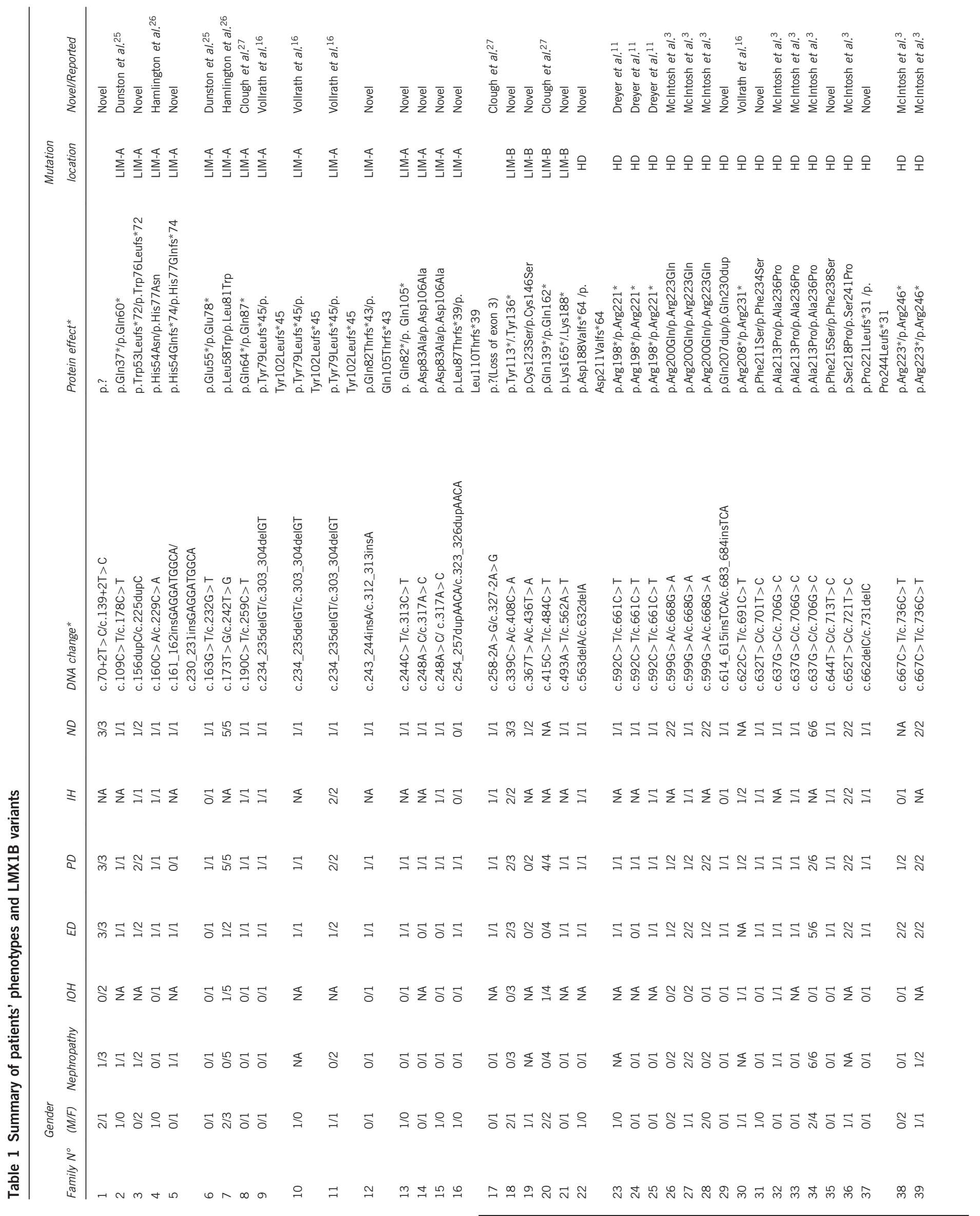




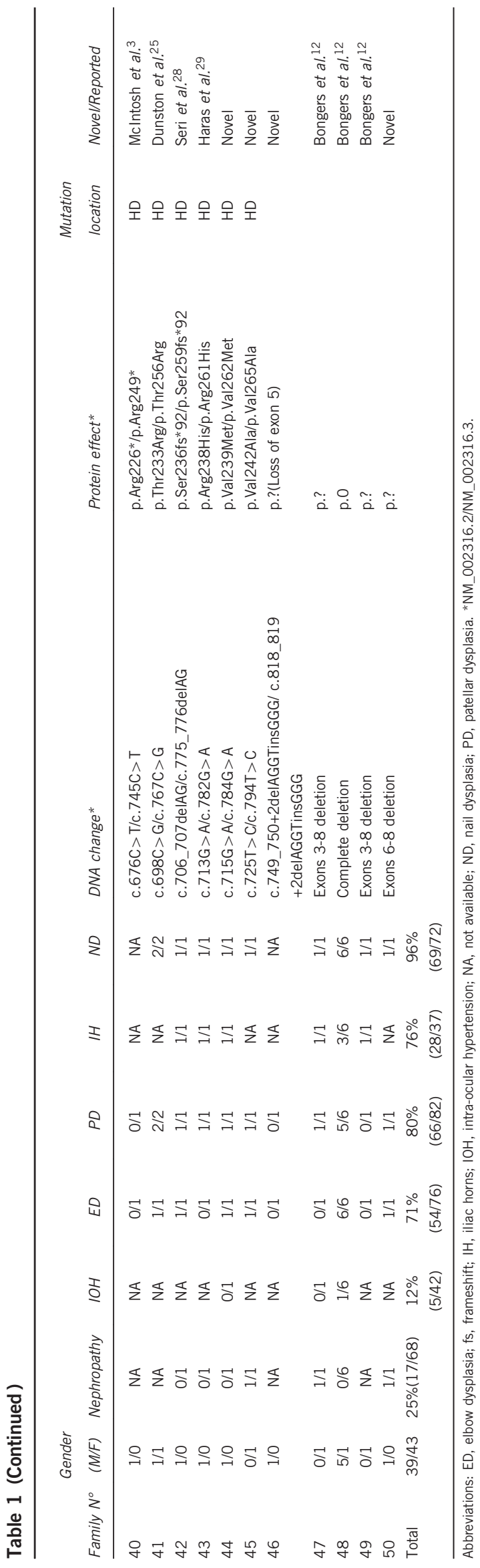

\section{MATERIAL AND METHODS}

\section{Patients}

We studied 94 subjects affected with NPS, 45 males and 49 females. Among them, 33 were sporadic cases and 61 individuals were members of 22 unrelated families (Table 1). Patients' samples were sent to our laboratory for $L M X 1 B$ molecular analysis. Most of them were recruited by clinical geneticists ( 48 families), a few by dermatologists (2 families), paediatricians (2 families), nephrologists (2 families) and orthopaedic surgeons (1 family). The great majority were of French origin (all geographic areas), while some cases were referred from Belgium, Switzerland, Spain, Australia and USA. Clinical data were obtained through detailed forms (describing skeletal, nail, renal and ocular features), completed by patients' referring physicians. Renal anomalies were defined as urinary total protein $>0.1 \mathrm{~g} / \mathrm{l}$ (or $>0.300 \mathrm{~g} / 24 \mathrm{~h}$ ) or as renal insufficiency. Ocular involvement was defined by presence of $\mathrm{IOH}(>20 \mathrm{~mm} \mathrm{Hg}$ ), glaucoma or normal tension glaucoma. Patients' consents were obtained prior to molecular analysis.

\section{Molecular analysis}

Sanger sequencing of exons. LMX1B analysis was conducted on genomic DNA extracted from peripheral blood samples using the EZ1 DNA blood kit with the BioRobot EZ1 (Qiagen, Courtaboeuf, France). The eight exons of the $L M X 1 B$ gene and their flanking intronic regions were amplified by PCR and directly sequenced on an ABI310 genetic analyzer (Applied Biosystems, Courtaboeuf, France). All primer sequences and reaction conditions are available on request. All variants were described according to the nomenclature GenBank accession number NM_002316.3. In order to compare with previous literature data, the correspondence with NM_002316.2 is indicated in Table 1. Novel variants have been submitted to LOVD database (http://databases.lovd.nl/shared/genes/LMX1B, submission ID: LMX1B_000145 to LMX1B_000159).

Multiplex ligation-dependant probe amplification (MLPA). If no variant was found by exon sequencing, MLPA analysis was carried out in order to identify large deletions/duplications, according to the manufacturer's protocol (MRC-Holland SALSA MLPA probe set P289, http://www.mlpa.com). MRC Holland Coffalyser Software was used to analyse the results.

Array-based comparative genomic hybridization (CGH). If no variant was found by exon sequencing and MLPA, genome-wide analysis of DNA copy number changes was performed using the Agilent Human Genome CGH microarray 244 K (Agilent Technologies, Santa Clara, CA, USA) according to the protocol provided by the manufacturer. Analysis of the data was performed with Agilent DNA Analytics 4.0 software. Classification as gain or loss was based on identification by the CGH plotter and visual inspection of the $\log _{2}$ ratios, using $z$-score and ADM-2 algorithms.

Genomic sequencing of whole LMX1B. When exon sequencing, MLPA and array-CGH were negative, we performed genomic sequencing of whole $L M X 1 B$ gene sequencing in order to explore the non-coding regions (promoter, 5'UTR, introns and 3'UTR): ch9.hg19: g.129,374,658_129,466,967. We designed 15 long-range PCRs of approximately $10 \mathrm{~kb}$ covering almost entirely the $L M X 1 B$ gene, for pyrosequencing using GS454 Junior (Roche, Basel, Switzerland) technology. The sequencing reads were aligned against the human chromosome 9 reference sequence (NC_000009.11) using GenSearch Software (PhenoSystems, Wallonia, Belgium). Variant calling was performed with the following parameters: at least $5 \times$ coverage, 


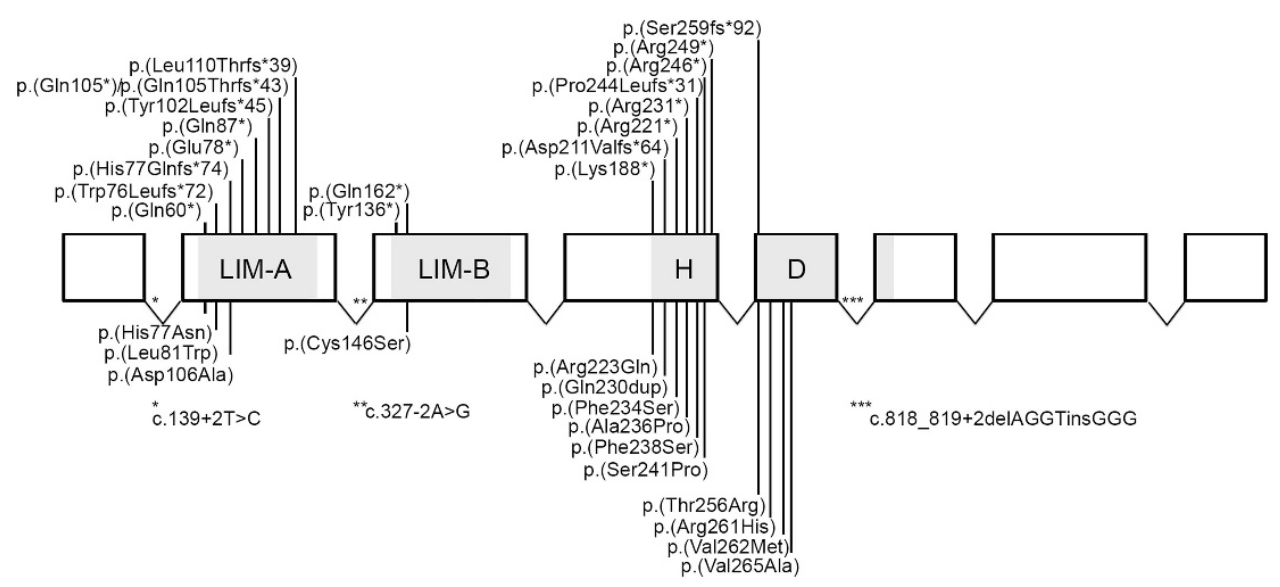

Figure 1 Schematic representation of $L M X 1 B$ variants. $L M X 1 B$ is composed of eight exons. The LIM-A and LIM-B domains are encoded by exons 2 and 3 , respectively, and the HD is encoded by exons 4 to 6 . Variants are clustered in these functional domains. Truncating variants are represented above the gene whereas missense variants are represented under the gene. Splicing variants are represented by stars.

minimal variant frequency $>10 \%$. Regions uncovered or with less than $10 \times$ depth coverage were Sanger-sequenced.

\section{RESULTS}

Molecular analyses

The first $L M X 1 B$ screening (ie Sanger sequencing of exons and MLPA) revealed a molecular anomaly in 50 families (91\%). We identified truncating variants in 31 families (48 patients); of these, 4 families were carriers of large deletions. Missense variants in LIM domains were found in 5 families (10 patients) and missense variants in HD domain in 14 families (24 patients) (Figure 1, Table 1). All variants occurred in LMX1B functional domains, that is, HD, LIM-A and LIMB domains. Several DNA changes were recurrent in our cohort: c.303_304delGT (p. $\left(\right.$ Tyr102Leufs $\left.\left.{ }^{\star} 45\right)\right), \quad$ c.661C $>$ T $\quad\left(p .\left(\operatorname{Arg} 221^{\star}\right)\right)$, c.668G $>$ A (p.(Arg223Gln)), c.706G $>$ C (p.(Ala236Pro)) and c.736C $>\mathrm{T}\left(\mathrm{p} .\left(\operatorname{Arg} 246^{*}\right)\right)$ identified in three families (Table 1). We identified six novel missense variants in our series: c.784G $>$ A (p.(Val262Met)) occurred de novo in a sporadic case (Family 44), c.436T > A (p.(Cys146Ser)) segregates with the phenotype in Family 19. Sample relationships were confirmed for the de novo variant. Parental studies were not available for the other four missense variants: c.317A $>$ C (p.(Asp106Ala)) (Families 14 and 15), c.701T $>C$ (p.(Phe234Ser)) (Family 31), c.713T $>$ C (p.(Phe238Ser)) (Family 35), c.794T $>$ C (p.(Val265Ala)) (Family 45). Finally, we identified a novel in-frame insertion c.683_684insTCA (p.(Gln230dup)) in Family 29, but parental studies were not available.

In five families, no molecular anomaly was identified in the LMX1B's eight exons and no large rearrangements were detected by array-CGH. We therefore performed genomic sequencing of whole LMX1B gene, including the non-coding regions, in families 51 to 54 (DNA material was insufficient for family 55). On average, 450 variants per patient were identified in $L M X 1 B$, most of them being located in intron 2 which spans approximately $75 \mathrm{~kb}$. We filtered: (i) the homozygous variants, assuming the autosomal dominant mode of inheritance of NPS in these families; (ii) the recurrent variants identified on control samples; and (iii) the single nucleotide polymorphisms reported in dbSNP (v135) (http:/www.ncbi.nlm.nih.gov/ projects/SNP) or 1000 Genomes Project database (http://www.1000 genomes.org/ ) with a minor allele frequency $>5 \%$. The remaining 64 variants (22 in family 51, 7 in family 52, 20 in family 53 and 15 in family 54) were studied by Sanger sequencing. Familial segregation was assessed when possible (families 51 and 52), and 100 control chromosomes were screened for the validated variants. Finally, only two variants of uncertain significance were remaining after the filtering (ch9.hg19:g.129,413,679delG in family 52 and ch9.hg19: g.129,437,509G $>\mathrm{T}$ in family 54), both being located in intron 2 at long-distance from the exon-intron boundaries (Supplementary Figure 3).

Clinical findings in the $\mathbf{5 0}$ families with $L M X 1 B$ variant/deletion Skeletal findings. In our cohort, $89 \%(n=73 / 82)$ patients presented with skeletal involvement (patella, scapula, elbow or pelvis) (Figure 2, Table 2). Eighty percent of patients $(n=66 / 82)$ presented with bilateral patellar involvement. Patellar hypoplasia was reported in $61 \%$ of the patients $(n=50 / 82)$ and patellar agenesis in $16 \%(n=13-$ /82). Subluxed patellae were observed in 32\% (26/82). Elbow dysplasia was present in $71 \%$ of the patients $(n=54 / 76)$, corresponding to elbow-limited extension in 59\% (45/76), radial subluxation in $26 \%$ (20/76) and radial head anomalies in 16\% (12/76). Only 37 patients had pelvis X-rays revealing iliac horns in $76 \%(n=28 / 37)$. Scapula involvement (thickening of the lateral border of the scapula, glenoid hypoplasia or limitation of shoulder motion) was rare, only identified in $9 \%$ of the patients $(n=7 / 82)$.

Nail involvement. Through the clinical form, we obtained information about nail involvement for $88 \%$ of the patients $(n=72 / 82)$ (Figure 2, Table 2). Most patients harboured nail anomalies (96\%, $n=69 / 72$ ). Koilonychia and longitudinal striation of nails were the most frequent defects, observed in $36 / 72$ patients (50\%). Anonychia was identified in $19 / 72$ patients (26\%) and affected mainly the thumbs. Lunulae anomalies comprised triangular lunulae (31/72, $29 \%$ ) or even absent lunulae $(9 / 72,13 \%)$, affecting predominantly the first and second digits.

Renal assessments. Renal investigations, quantitative urinalysis and creatinine clearance measurements were obtained in $80 \%$ of the patients $(n=68 / 82)$, aged 3-64 years (median 29). Proteinuria was identified in $25 \%(n=17 / 68)$. Three patients from family 34 had endstage renal failure (Table 1).

Prevalence of nephropathy increased with age. We observed that $12.5 \%(n=3 / 24)$ of affected patients were under 15 years whereas $32 \% \quad(n=14 / 44)$ were over 15 years. There was no statistically 
a
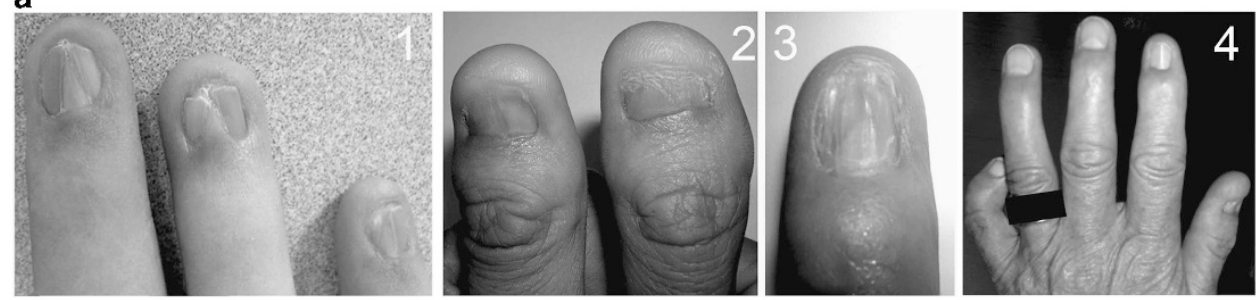

b
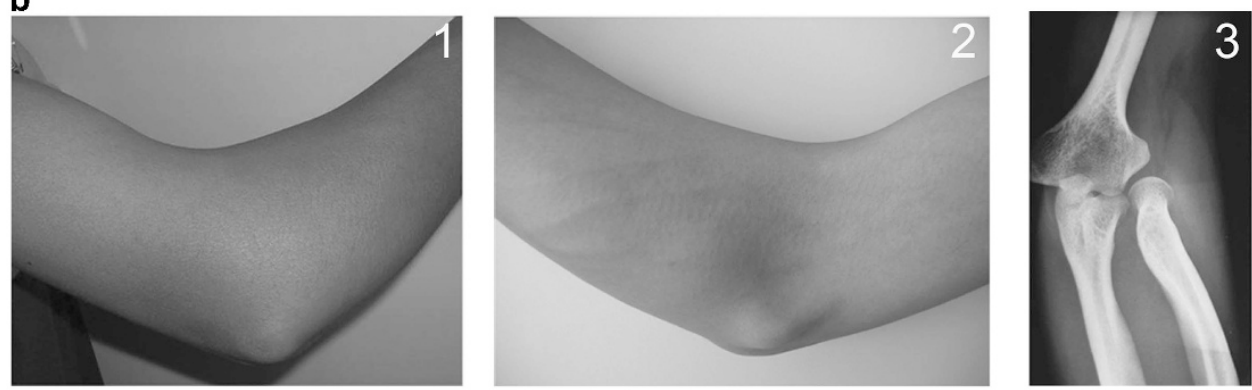

C
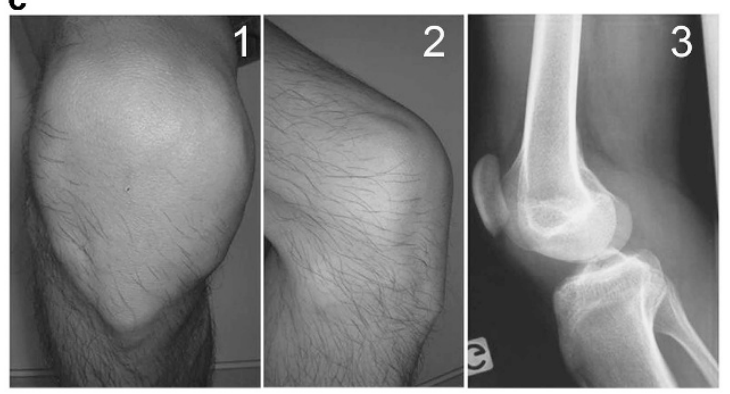

d

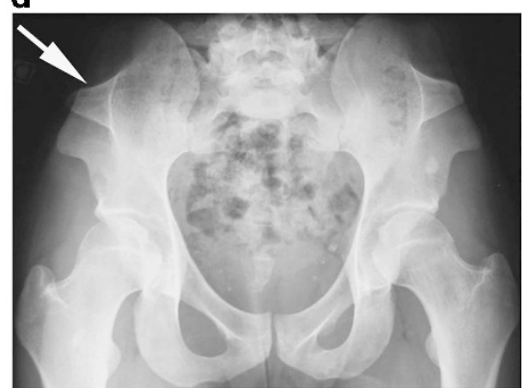

Figure 2 Clinical features in NPS patients. (a) Nail dysplasia. (1) Family 12, nails show more severe dysplasia on the ulnar side. (2, 3) Family 45, thumbnail dysplasia and koilonychia of the second digit in the same patient. (4) Family 48, nail dysplasia of the first and second digits. Typical triangular lunula of the third digit. (b) Elbow dysplasia. (1, 2) Family 43, limitation of the elbow extension. (3) Elbow X-ray showing dysplastic dislocated radial head and hypoplasia of the capitellum. (c) Patellar dysplasia. $(1,2)$ Family 43, subluxed patella on knee flexion. (3) Family 43, knee X-ray showing superior displacement of hypoplastic patella. (d) Family 43, pelvis X-ray showing typical iliac horns (arrow).

significant gender difference: $19 \%(n=6 / 31)$ of affected patients were males versus $30 \%(n=11 / 37)$ females $(P=0.48$, adjusted for age).

Ophthalmological assessments. In our cohort, 52\% of the patients ( $n=43 / 82)$ had intra-ocular pressure measurements. IOH was identified in $21 \%$ of them $(n=9 / 43)$. Among these nine patients, six carried a truncating variant, two presented with a missense variant in LIM-A and one with a missense variant in HD (Table 1). Five were younger than 40 years.

Clinical findings in the five families without $L M X 1 B$ anomaly Pedigrees and clinical findings in the five families without $L M X 1 B$ anomaly are depicted in Supplementary Figure 1 and summarised in Supplementary Table 1. These families were evaluated by clinical geneticists and considered typical of NPS. The frequency of nail, elbow and patellar dysplasia, ocular and renal involvement was not statistically different from that observed in the series of patients harbouring an $L M X 1 B$ alteration. Regarding the pelvis dysplasia (observed in $76 \%$ of patients with $L M X 1 B$ alteration), only three patients had pelvisXrays revealing no iliac horns.

\section{DISCUSSION}

$L M X 1 B$ is the major gene responsible for NPS. Indeed, $91 \%$ of patients in our series of 55 families harbour a variant/deletion

\section{Table 2 Prevalence of nail and skeletal dysplasia in NPS patients}

\begin{tabular}{lccc}
\hline & \multicolumn{3}{c}{ Frequency (\%) } \\
& Present study & Reported patients & Total \\
\hline Nail dysplasia & $96 \%(69 / 72)$ & $99 \%(299 / 302)$ & $98 \%(368 / 374)$ \\
$\quad$ Anonychia & $26 \%(19 / 72)$ & & \\
Koilonychia & $50 \%(36 / 72)$ & & \\
Triangular lunulae & $29 \%(21 / 72)$ & & \\
Patellar dysplasia & $80 \%(66 / 82)$ & $90 \%(229 / 253)$ & $88 \%(295 / 335)$ \\
$\quad$ Patellar hypoplasia & $61 \%(50 / 82)$ & & \\
Patellar agenesis & $16 \%(13 / 82)$ & & \\
Patellar dislocation & $32 \%(26 / 82)$ & & \\
Elbow dysplasia & $71 \%(54 / 76)$ & $84 \%(223 / 266)$ & $81 \%(277 / 342)$ \\
$\quad$ Extension limitation & $59 \%(45 / 76)$ & & \\
$\quad$ Radial head anomalies & $47 \%(36 / 76)$ & & \\
Iliac horns & $76 \%(28 / 37)$ & $79 \%(149 / 188)$ & $78 \%(177 / 225)$ \\
\hline
\end{tabular}

Literature patients: Knoers et al.; ${ }^{17}$ Lee et al.; ${ }^{20}$ Lin et al.; ${ }^{21}$ Milla et al.; ${ }^{22}$ Mimiwati et al.; ${ }^{23}$ Romero et al..$^{24}$

identified by routine diagnosis screening (Sanger sequencing of exons and MLPA). We identified 38 different $L M X 1 B$ variants or deletions in 50 index patients and their 32 relatives. About half of the variants were recurrent, identified in several families or already reported in the 
literature (Table 1). For most of them, a relationship between families or a founder effect is unlikely as they were of different ethnic origins and from different geographic areas. As previously reported, all identified variants clustered in the LIM and HD domains. ${ }^{3,16}$ Among the 38 different $L M X 1 B$ anomalies, 19 had not been reported before. Of these, 12 anomalies were considered clearly responsible for the disease as they were either frameshift, nonsense or splice variants. The remaining anomalies were six missense variants and one in-frame insertion. Missense variant c.784G $>$ A (p.(Val262Met)) occurred de novo in a sporadic case of NPS, and is therefore likely responsible for affected protein function. Missense variant c.436T > A (p.(Cys146Ser)) was found to segregate with NPS in the family, indicating that it is likely responsible for the disease. Inheritance studies were unfortunately not available for the other novel variants. All variants altered highly conserved nucleotides up to tetraodon (http://genome.ucsc. edu) and were predicted in silico to be probably (c.436T $>\mathrm{A}$, c.701T $>$ C, c.713T $>$ C, c.794T $>$ C) or possibly (c.317A $>$ C) damaging by Polyphen-2 V2.2.2 (http://genetics.bwh.harvard.edu/pph2/ index.shtml), and deleterious by SIFT software (http//sift.bii.a-star. edu.sg/). Missense c.317A $>C$ (p.(Asp106Ala)) was identified in two unrelated families from Spain, suggesting a founder effect.

Nail and skeletal phenotypes were consistent with the literature data (Table 2). ${ }^{17,20-24}$ Almost all patients with an $L M X 1 B$ variant/deletion presented with nail involvement (96\%). First and second digits were preferentially involved and anonychia, koilonychia, longitudinal striations, triangular and absent lunulae were observed. Patellar defects were identified in $86 \%$ and iliac horns in $76 \%$, which is comparable with previous reports. ${ }^{2,4}$ Upper limb defects (66\%) were less frequent than lower limb anomalies (86\%).

Renal involvement is the major determinant of prognosis in NPS. We identified nephropathy in $25 \%$ of patients with an LMX1B variant/ deletion, usually consisting of proteinuria. In addition, we noted that all six individuals from family 34 (missense variant in HD c.706G > C, p.(Ala236Pro)) presented with nephropathy, and three of them were severely affected with end-stage renal failure. This recurrent variant was identified in two other sporadic cases from our cohort (families 32 and 33): the case from family 32 had nephropathy when examined at age 33 , while the case from family 33 had no renal disease at age 5 . The variant had also been reported in four previous families. ${ }^{2,3,17}$ Interestingly, four of the five patients reported in the literature had nephropathy, suggesting a high prevalence of renal involvement associated with this variant. This was consistent with previous studies showing that the p.(Ala236Pro) mutant has a statistically significant lower contribution to wild-type LMX1B transactivation compared with other mutants. ${ }^{13}$

Ophthalmological anomalies were rarely reported in previous studies. $^{2,4,18}$ In our cohort, $20 \%$ of patients with an $L M X 1 B$ variant/ deletion presented with either IOH or glaucoma.

NPS patients showed high inter-individual and intra-familial variability, stressing the possible involvement of modifier genes in the expression of the phenotype. Through the clinical form completed by the referring physicians, we realised that patients were inadequately followed up, because most of them had no renal or ophthalmological input. In agreement with previous recommendations for clinical management, ${ }^{2,4}$ we stress the need for renal and ophthalmological follow-up in NPS patients. The annual renal screening (blood pressure and urinanalysis) should be performed soon after birth, especially if a variant in $L M X 1 B$ HD has been identified. ${ }^{2}$ The ophthalmological examination (intra-ocular pressure measurement, optic disc examination and visual field assessment) should be performed every 2 years in any adult affected with NPS.
In $9 \%$ of the families tested, no alteration was found in $L M X 1 B$ by the routine screening. These patients were not clinically different from the patients harbouring an $L M X 1 B$ alteration. Analyses were pursued in four families by next-generation genomic sequencing of whole $L M X 1 B$ gene. Only two variants located within intron 2 at longdistance from the exon-intron boundaries were remaining after the filtering: ch9.hg19:g.129,413,679delG in family 52 and ch9.hg19: g.129,437,509G $>$ T in family 54. These variants have never been described. They affect nucleotides that are partly conserved among species but are not lying within an evolutionary conserved region (Supplementary Figure 3). No binding site for transcription factors is predicted in silico according to the JASPAR software (http://jaspardev. genereg.net/). For both variants, in silico splicing predictions using MaxEntScan software ${ }^{19}$ do not show the creation of a cryptic splice site. Therefore, we considered that these variants are likely to be rare non-pathogenic variants. Owing to the large size of the intron 2 which spans approximately $75 \mathrm{~kb}$, an ex vivo splicing assay would be difficult to design to confirm this conclusion.

For family 51, segregation analysis was performed based on variants generated by next-generation sequencing and microsatellite markers (Supplementary Figure 2). Interestingly, no linkage to $L M X 1 B$ locus (9q33.3) was shown, suggesting that a genomic alteration of this gene is not involved for this particular family. In the other families, the number of available individuals was insufficient for linkage analysis.

To our knowledge, the hypothesis of a genetic heterogeneity has never been studied in NPS. Given the large number of genes involved in the WNT signaling pathway, potential candidate genes are numerous. $L M X 1 B$ deregulation due to a genomic alteration in another locus could also be a hypothesis. Quantitative expression studies are difficult to achieve because of very low LMX1B mRNA expression in readily accessible tissues (lymphoblasts, fibroblasts). Therefore, approach by whole genome studies should be considered in these five families with no $L M X 1 \mathrm{~B}$ alteration.

\section{CONFLICT OF INTEREST}

The authors declare no conflict of interest.

\section{ACKNOWLEDGEMENTS}

We gratefully acknowledge all families and clinicians who participated to this study: M Abramowicz, MC Addor, Antignac C, Y Alembick, JL Alessandri, C Baumann, P Blanchet, S Blesson, F Boralevi, A Boutemy, F Bridoux, I Citony-Garnon, E Colin, P Collignon, V Cormier-Daire, Dallocchio, A David, P Edery, C Francannet, C Goizet, A Hovnanian, D Lacombe, M Le Merrer, M Lebrun, B Leheup, JY Lespinasse, D Martin-Coignard, L Martorell-Sampol, G Morin, L Oprea, N Philip, M Rossi, S Sandaradura, P Sarda, S Sigaudy, $\mathrm{M}$ Teboul, J Vigneron, A Zankl. We also thank E Thomas for critical reading of the manuscript, and MP Delescaut and O Fruchard for their invaluable technical assistance.

1 Roeckerath W: [Hereditary osteo-onychodysplasia]. Fortschr Geb Rontgenstr 1951; 75: 700-712.

2 Bongers EM, Huysmans FT, Levtchenko E et al: Genotype-phenotype studies in nailpatella syndrome show that LMX1B mutation location is involved in the risk of developing nephropathy. Eur J Hum Genet 2005; 13: 935-946.

3 Mclntosh I, Dreyer SD, Clough MV et al: Mutation analysis of LMX1B gene in nailpatella syndrome patients. Am J Hum Genet 1998; 63: 1651-1658.

4 Sweeney E, Fryer A, Mountford R, Green A, McIntosh I: Nail patella syndrome: a review of the phenotype aided by developmental biology. J Med Genet 2003; 40: 153-162.

5 Lichter PR, Richards JE, Downs CA, Stringham HM, Boehnke M, Farley FA: Cosegregation of open-angle glaucoma and the nail-patella syndrome. Am J Ophthalmol 1997; 124: 506-515.

6 Bennett WM, Musgrave JE, Campbell RA et al: The nephropathy of the nail-patella syndrome. Clinicopathologic analysis of 11 kindred. Am J Med 1973; 54: 304-319. 
7 Granata A, Nori G, Ravazzolo R et al: Nail-patella syndrome and renal involvement. Description of three cases and literature review. Clin Nephrol 2008; 69: 377-382.

8 McIntosh I, Dunston JA, Liu L, Hoover-Fong JE, Sweeney E: Nail patella syndrome revisited: 50 years after linkage. Ann Hum Genet 2005; 69: 349-363.

9 Meyrier A, Rizzo R, MC G: The nail-patella syndrome, a review. J Nephrol 1990; 2. 133-140.

10 Chen $\mathrm{H}$, Lun Y, Ovchinnikov $\mathrm{D}$ et al: Limb and kidney defects in Lmx1b mutant mice suggest an involvement of LMX1B in human nail patella syndrome. Nat Genet 1998; 19: 51-55.

11 Dreyer SD, Zhou G, Baldini A et al: Mutations in LMX1B cause abnormal skeletal patterning and renal dysplasia in nail patella syndrome. Nat Genet 1998; 19: 47-50.

12 Bongers EM, de Wijs IJ, Marcelis C, Hoefsloot LH, Knoers NV: Identification of entire LMX1B gene deletions in nail patella syndrome: evidence for haploinsufficiency as the main pathogenic mechanism underlying dominant inheritance in man. Eur J Hum Genet 2008; 16: 1240-1244.

13 Dreyer SD, Morello R, German MS et al: LMX1B transactivation and expression in nail-patella syndrome. Hum Mol Genet 2000; 9: 1067-1074.

14 Sato U, Kitanaka S, Sekine T, Takahashi S, Ashida A, Igarashi T: Functional characterization of LMX1B mutations associated with nail-patella syndrome. Pediatr Res 2005; 57: 783-788.

15 Cross SH, Macalinao DG, McKie L et al: A dominant-negative mutation of mouse Lmx1b causes glaucoma and is semi-lethal via LBD1-mediated dimerisation. PLoS Genet 2014; 10: e1004359.

16 Vollrath D, Jaramillo-Babb VL, Clough MV et al: Loss-of-function mutations in the LIMhomeodomain gene, LMX1B, in nail-patella syndrome. Hum Mol Genet 1998; 7: 1091-1098.

17 Knoers NV, Bongers EM, van Beersum SE, Lommen EJ, van Bokhoven H, Hol FA: Nailpatella syndrome: identification of mutations in the LMX1B gene in Dutch families. J Am Soc Nephrol 2000; 11: 1762-1766.
18 Pressman $\mathrm{CL}$, Chen $\mathrm{H}$, Johnson RL: LMX1B, a LIM homeodomain class transcription factor, is necessary for normal development of multiple tissues in the anterior segment of the murine eye. Genesis 2000; 26: 15-25.

19 Yeo G, Burge CB: Maximum entropy modeling of short sequence motifs with applications to RNA splicing signals. J Comput Biol 2004; 11: 377-394.

20 Lee BH, Cho TJ, Choi HJ et al: Clinico-genetic study of nail-patella syndrome. J Korean Med Sci 2009; 24: S82-S86.

21 Lin Y, Zhao J, Chen S et al: A novel mutation in LMX1B gene causes nail-patella syndrome in a large Chinese family. Bone 2008; 43: 591-595.

22 Milla E, Hernan I, Gamundi MJ, Martinez-Gimeno M, Carballo M: Novel LMX1B mutation in familial nail-patella syndrome with variable expression of open angle glaucoma. Mol Vis 2007; 13: 639-648.

23 Mimiwati Z, Mackey DA, Craig JE et al: Nail-patella syndrome and its association with glaucoma: a review of eight families. Br J Ophthalmol 2006; 90: 1505-1509.

24 Romero P, Sanhueza F, Lopez P, Reyes L, Herrera L: C.194A >C (Q65P) mutation in the LMX1B gene in patients with nail-patella syndrome associated with glaucoma. Mol Vis 2011; 17: 1929-1939.

25 Dunston JA, Hamlington JD, Zaveri J et al: The human LMX1B gene: transcription unit, promoter, and pathogenic mutations. Genomics 2004; 84: 565-576.

26 Hamlington JD, Jones C, McIntosh I: Twenty-two novel LMX1B mutations identified in nail patella syndrome (NPS) patients. Hum Mutat 2001; 18: 458.

27 Clough MV, Hamlington JD, Mclntosh I: Restricted distribution of loss-of-function mutations within the LMX1B genes of nail-patella syndrome patients. Hum Mutat 1999; 14: 459-465.

28 Seri M, Melchionda S, Dreyer S et al: Identification of LMX1B gene point mutations in italian patients affected with Nail-Patella syndrome. Int J Mol Med 1999; 4: 285-290.

29 Haras B, Vulpoi F, Onose G: A case of nail-patella syndrome associated with thyrotoxicosis. J Med Life 2012; 5: 126-129.

Supplementary Information accompanies this paper on European Journal of Human Genetics website (http://www.nature.com/ejhg) 